Supplement of Biogeosciences, 16, 4307-4320, 2019

https://doi.org/10.5194/bg-16-4307-2019-supplement

(C) Author(s) 2019. This work is distributed under

the Creative Commons Attribution 4.0 License.

(c) (1)

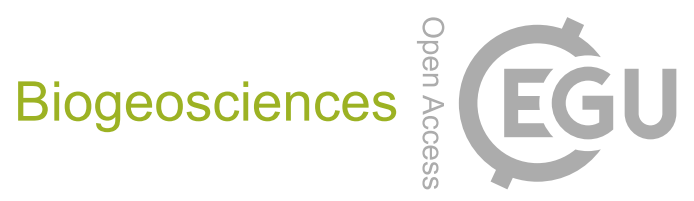

Supplement of

\title{
Organic matter contents and degradation in a highly trawled area during fresh particle inputs (Gulf of Castellammare, southwestern Mediterranean)
}

Sarah Paradis et al.

Correspondence to: Sarah Paradis (sarah.paradis@uab.cat)

The copyright of individual parts of the supplement might differ from the CC BY 4.0 License. 


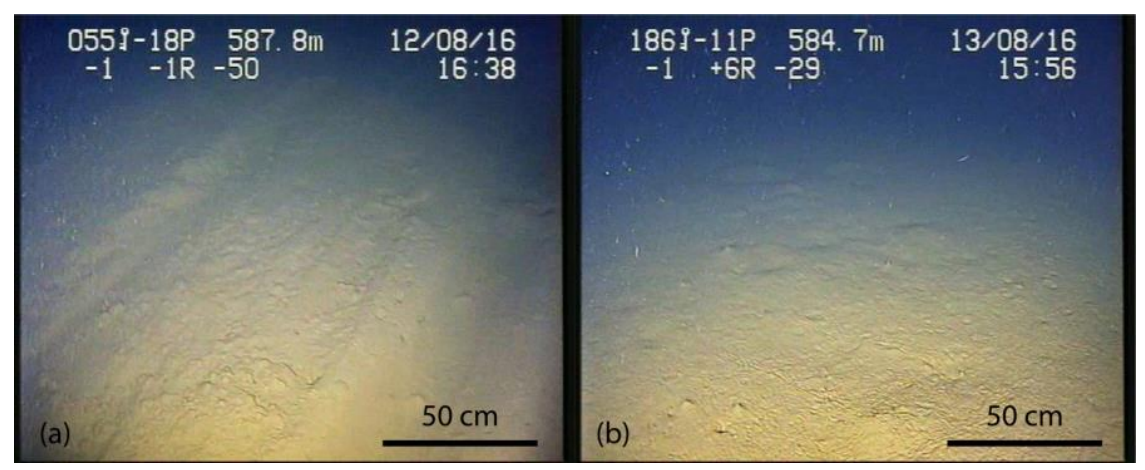

Figure S1. ROV-seafloor pictures from the trawled (a) and untrawled (b) sites in the Gulf of Castellammare. The trawled site presents linear marks and furrows on eroded sediments produced by the intense trawling activity in the area, whereas the untrawled site shows mounds and tracks characteristic of bioturbation activity.
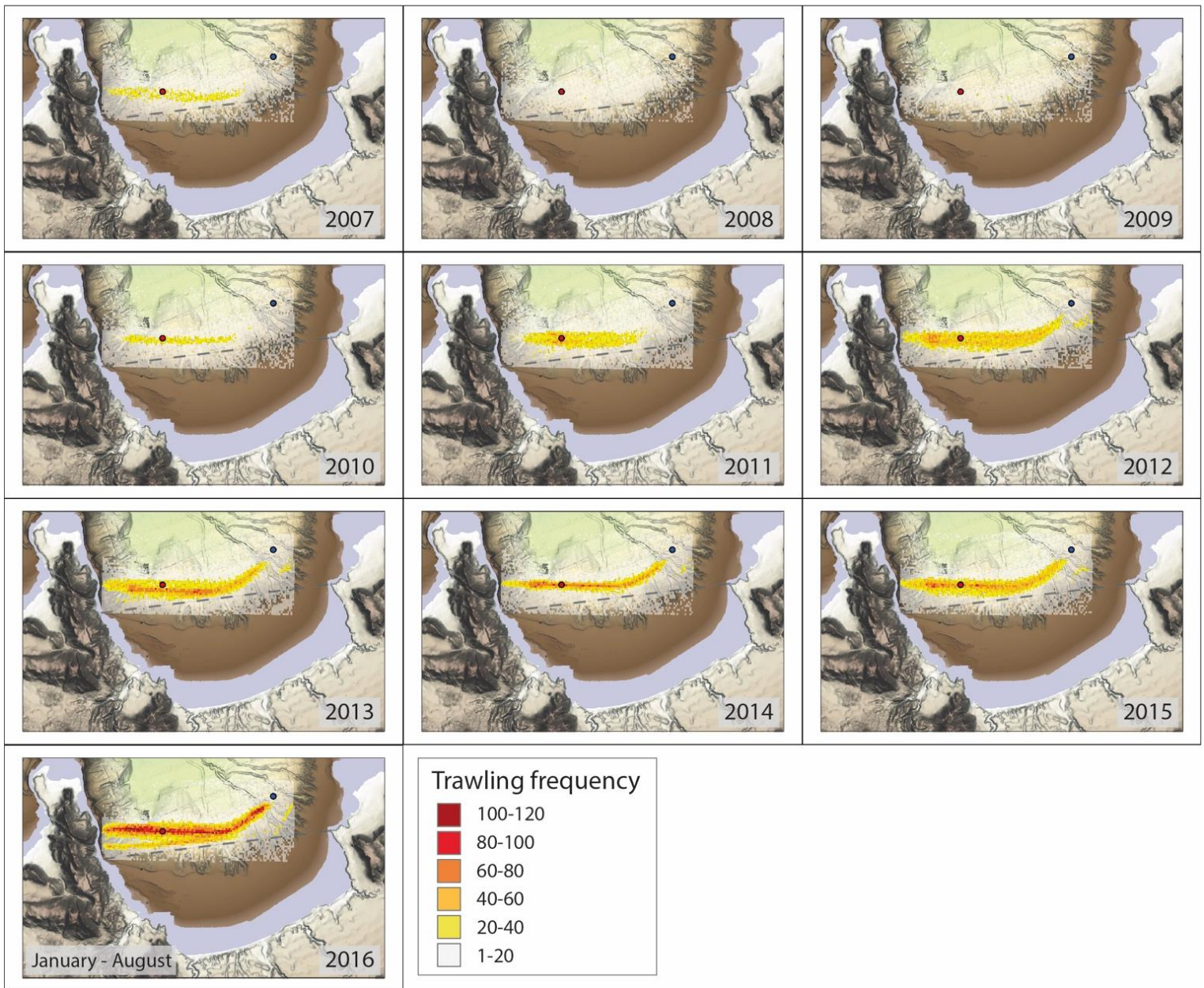

Figure S2. Evolution of trawling intensity. Number of times trawled per year and per grid cell (200 x $200 \mathrm{~m})$ in the Gulf of Castellammare. Note the increasing trawling frequency since 2007, with the exception of 2008 and 2009, and that computation of trawling frequency in 2016 is limited to VMS data prior to the sampling period, August $10^{\text {th }}$. Blue and red circles indicate the sampling sites of the untrawled, and trawled site, respectively. 
Table S1. Results of the PERMANOVA test for differences in organic matter quantity (protein, carbohydrate, lipid, biopolymeric C, and phytopigment) between trawled and untrawled sites, depth layers, and their interaction. The percentage variance explained by each source of variation is also reported. $\mathrm{df}=$ degrees of freedom; $\mathrm{MS}=$ mean square; Pseudo-F = permutational $\mathrm{F}$; significance of test obtained from Monte Carlo simulations: $*=p(\mathrm{MC})<0.05 ; * *=p(\mathrm{MC})<0.01 ; * * *=p(\mathrm{MC})<0.001 ; \mathrm{n} . \mathrm{s} .=$ not significant.

\begin{tabular}{|c|c|c|c|c|c|c|}
\hline Variable & Source & df & MS & Pseudo-F & $p(\mathrm{MC})$ & Explained variance $(\%)$ \\
\hline \multirow[t]{5}{*}{ Protein $\left(\mathrm{mgC} \cdot \mathrm{g}^{-1}\right)$} & Site & 1 & 4.62 & 13.2 & $* *$ & 22 \\
\hline & Depth & 4 & 3.51 & 10.1 & $* * *$ & 40 \\
\hline & Site $\mathrm{x}$ Depth & 4 & 0.82 & 2.4 & n.s. & 12 \\
\hline & Residual & 20 & 0.35 & & & 27 \\
\hline & Total & 29 & & & & \\
\hline \multirow[t]{5}{*}{ Carbohydrate $\left(\mathrm{mgC} \cdot \mathrm{g}^{-1}\right)$} & Site & 1 & 11.29 & 47.9 & $* * *$ & 44 \\
\hline & Depth & 4 & 1.68 & 7.1 & $* *$ & 15 \\
\hline & Site $x$ Depth & 4 & 1.57 & 6.6 & $* *$ & 27 \\
\hline & Residual & 20 & 0.23 & & & 14 \\
\hline & Total & 29 & & & & \\
\hline \multirow[t]{5}{*}{ Lipid $\left(m g C \cdot g^{-1}\right)$} & Site & 1 & 4.68 & 20.4 & $* * *$ & 23 \\
\hline & Depth & 4 & 4.73 & 20.6 & $* * *$ & 59 \\
\hline & Site x Depth & 4 & 0.20 & 0.9 & n.s. & 0 \\
\hline & Residual & 20 & 0.23 & & & 18 \\
\hline & Total & 29 & & & & \\
\hline \multirow[t]{5}{*}{ Biopolymeric $\mathrm{C}\left(\mathrm{mgC} \cdot \mathrm{g}^{-1}\right)$} & Site & 1 & 13.02 & 36.9 & $* * *$ & 55 \\
\hline & Depth & 4 & 1.36 & 3.9 & $*$ & 11 \\
\hline & Site $\mathrm{x}$ Depth & 4 & 0.87 & 2.5 & n.s. & 11 \\
\hline & Residual & 20 & 0.35 & & & 23 \\
\hline & Total & 29 & & & & \\
\hline \multirow[t]{5}{*}{ Phytopigment $\left(\mu \mathrm{gC} \cdot \mathrm{g}^{-1}\right)$} & Site & 1 & 11.25 & 93.0 & $* * *$ & 50 \\
\hline & Depth & 4 & 3.53 & 29.1 & $* * *$ & 38 \\
\hline & Site $x$ Depth & 4 & 0.29 & 2.4 & n.s. & 4 \\
\hline & Residual & 20 & 0.12 & & & 8 \\
\hline & Total & 29 & & & & \\
\hline
\end{tabular}


Table S2. Results of the pairwise comparison testing for differences in the quantity of each investigated variable between trawled and untrawled sites at each depth. $\mathrm{df}=$ degrees of freedom; MS = mean square; Pseudo-F = permutational F; significance of test obtained from Monte Carlo simulations: $*=p(\mathrm{MC})<0.05 ; * *=p(\mathrm{MC})<0.01 ; * * *=p(\mathrm{MC})<0.001$; n.s. $=$ not significant.

Pair-wise comparisons

Factor: Trawled vs. Untrawled

\begin{tabular}{|c|c|c|c|}
\hline Variable & Depth $(\mathrm{cm})$ & $\mathrm{t}$ & $p(\mathrm{MC})$ \\
\hline \multirow[t]{5}{*}{ Protein $\left(\mathrm{mgC} \cdot \mathrm{g}^{-1}\right)$} & $0-1$ & 0.502 & n.s. \\
\hline & $1-3$ & 1.236 & n.s. \\
\hline & $3-5$ & 1.871 & n.s. \\
\hline & $5-7$ & 2.94 & $*$ \\
\hline & $7-9$ & 1.799 & n.s. \\
\hline \multirow[t]{5}{*}{ Carbohydrate $\left(\mathrm{mgC} \cdot \mathrm{g}^{-1}\right)$} & $0-1$ & 3.648 & $*$ \\
\hline & $1-3$ & 9.139 & $* *$ \\
\hline & $3-5$ & 2.997 & $*$ \\
\hline & $5-7$ & 0.643 & n.s. \\
\hline & $7-9$ & 1.06 & n.s. \\
\hline \multirow[t]{5}{*}{ Lipid $\left(\mathrm{mgC} \cdot \mathrm{g}^{-1}\right)$} & $0-1$ & 0.666 & n.s. \\
\hline & $1-3$ & 3.753 & $*$ \\
\hline & $3-5$ & 1.044 & n.s. \\
\hline & $5-7$ & 2.921 & $*$ \\
\hline & $7-9$ & 5.63 & $* *$ \\
\hline \multirow[t]{5}{*}{ Biopolymeric C $\left(\mathrm{mgC} \cdot \mathrm{g}^{-1}\right)$} & $0-1$ & 2.058 & n.s. \\
\hline & $1-3$ & 4.197 & $*$ \\
\hline & $3-5$ & 2.737 & n.s. \\
\hline & $5-7$ & 3.105 & $*$ \\
\hline & $7-9$ & 3.783 & $*$ \\
\hline \multirow[t]{5}{*}{ Phytopigment $\left(\mu \mathrm{gC} \cdot \mathrm{g}^{-1}\right)$} & $0-1$ & 1.846 & n.s. \\
\hline & $1-3$ & 6.128 & $* *$ \\
\hline & $3-5$ & 11.417 & $* * *$ \\
\hline & $5-7$ & 4.572 & $*$ \\
\hline & $7-9$ & 3.251 & $*$ \\
\hline
\end{tabular}

Table S3. Results of the PERMANOVA testing for differences in biochemical composition (protein, carbohydrate, lipid and phytopigment 5 contents) between trawled and untrawled sites, depth layers, and their interaction. The percentage variance explained by each source of variation is also reported. $\mathrm{df}=$ degrees of freedom; $\mathrm{MS}=$ mean square; Pseudo-F = permutational F; significance of test obtained from Monte Carlo simulations: $*=p(\mathrm{MC})<0.05 ; * *=p(\mathrm{MC})<0.01$; $* * *=p(\mathrm{MC})<0.001$; n.s. $=$ not significant.

\begin{tabular}{lllllll} 
& Source & df & MS & Pseudo-F & $p(\mathrm{MC})$ & Explained variance $(\%)$ \\
\hline Biochemical composition & Site & 1 & 31.9 & 34.0 & $* * *$ & 36 \\
& Depth & 1 & 13.5 & 14.4 & $* * *$ & 36 \\
& Site x Depth & 4 & 2.9 & 3.0 & $* *$ & 11 \\
& Residual & 20 & 0.9 & & & 16
\end{tabular}


Table S4. Results of the pairwise comparison testing for differences in the biochemical composition (in terms of protein. carbohydrate. lipid and phytopigment contents) between trawled and untrawled sites at each depth. $\mathrm{df}=$ degrees of freedom; MS = mean square; Pseudo-F = permutational F; significance of test obtained from Monte Carlo simulations: $*=p(\mathrm{MC})<0.05 ; * *=p(\mathrm{MC})<0.01 ; * * *=p(\mathrm{MC})<0.001$; n.s. $=$ not significant.

Pair-wise comparisons

Factors: Trawled vs.Untrawled

\begin{tabular}{llll} 
& Depth $(\mathrm{cm})$ & $\mathrm{t}$ & $p(\mathrm{MC})$ \\
\hline Biochemical composition & $0-1$ & 2.237 & n.s. \\
$1-3$ & 5.353 & $* *$ \\
$3-5$ & 2.456 & $*$ \\
$5-7$ & 2.565 & $*$ \\
$7-9$ & 2.875 & $*$
\end{tabular}

5

Table S5. Results of the PERMANOVA testing for differences in (a) relative contribution of phytopigments to biopolymeric C and (b) protein $\mathrm{C}$ turnover rates between examined factors and their interaction. The pairwise comparison testing for differences of each investigated variable between trawled and untrawled sites at each depth is also included. $\mathrm{df}=$ degrees of freedom; MS = mean square; Pseudo-F = permutational F; significance of test obtained from Monte Carlo simulations: $*=p(\mathrm{MC})<0.05 ; * *=p(\mathrm{MC})<0.01 ; * * *=p(\mathrm{MC})<0.001$;

10 n.s.= not significant.

(a)

Pair-wise comparisons

Factors: Trawled vs.Untrawled

\begin{tabular}{|c|c|c|c|c|c|c|c|c|c|}
\hline & Source & df & MS & Pseudo-F & $p(\mathrm{MC})$ & $\begin{array}{c}\text { Explained } \\
\text { variance }(\%)\end{array}$ & Depth $(\mathrm{cm})$ & $\mathrm{t}$ & $p(\mathrm{MC})$ \\
\hline \multirow{4}{*}{ Phytopigment/BPC (\%) } & Depth & 4 & 6 & 99.5 & $* * *$ & 77 & $1-3$ & 3.440 & $*$ \\
\hline & Site $\mathrm{x}$ Depth & 4 & 0.1 & 1.3 & n.s. & 0 & $3-5$ & 6.698 & $* *$ \\
\hline & Residual & 20 & 0.1 & & & 5 & $5-7$ & 4.143 & $*$ \\
\hline & Total & 29 & & & & 18 & 7-9 & 4.588 & $* *$ \\
\hline
\end{tabular}

(b)

Pair-wise comparisons

Factors: Trawled vs.Untrawled

\begin{tabular}{|c|c|c|c|c|c|c|c|c|c|}
\hline & Source & $\mathrm{df}$ & MS & Pseudo-F & $p(\mathrm{MC})$ & $\begin{array}{c}\text { Explained } \\
\text { variance }(\%)\end{array}$ & Depth $(\mathrm{cm})$ & $\mathrm{t}$ & $p(\mathrm{MC})$ \\
\hline \multirow{4}{*}{ Turnover $\left(10^{-3} \mathrm{~d}^{-1}\right)$} & Depth & 4 & 5.5 & 120.4 & $* * *$ & 64 & $1-3$ & 5.452 & $* *$ \\
\hline & Site x Depth & 4 & 1.2 & 26.4 & $* * *$ & 27 & $3-5$ & 4.242 & $*$ \\
\hline & Residual & 20 & 0 & & & 3 & $5-7$ & 1.234 & n.s. \\
\hline & Total & 29 & & & & 5 & $7-9$ & 2.418 & n.s. \\
\hline
\end{tabular}

